\title{
New Therapeutic Targets
}

\author{
Magali Provansal ${ }^{1}$, Maria Cappiello ${ }^{1}$, \\ Frederique Rousseau ${ }^{1}$, Anthony Goncalves ${ }^{1,2}$ and Patrice Viens ${ }^{1,2}$ \\ 1 Paoli-Calmettes Institute, Marseille \\ ${ }^{2}$ Faculty of Medicine of Marseille
}

France

\section{Introduction}

Cervical cancer occurs in approximately 500,000 women and kills 288,000 women worldwide each year. Prognosis is highly dependent on disease stage at diagnosis. When detected early, cervical cancer is generally curable. Early lesions are treated surgically and locally advanced lesions are managed with concurrent cisplatin chemotherapy and pelvic radiation. Unfortunately, responses to chemoradiation are partial and are of short duration.

Metastatic disease or recurrent lesions not amenable to radical local excision or regional radiation are treated with palliative chemotherapy. Current chemotherapeutic regimens are associated with significant side effects and only limited activity making the identification of active and tolerable novel targeted agents a high priority.

The evidence supporting the biological rational to combine novel non-cytotoxic agents with chemoradiotherapy is strong, and drugs targeting different molecular pathways are currently under clinical development (EGFR inhibitors, COX-2 inhibitors, hypoxia targeted agents, etc). Early pre-clinical and clinical strategies also favor the use of vascular-targeted agents with the aim to normalize the abnormal tumor vasculature, increase tumor oxygenation, and reduce interstitial fluid pressure. The integration of these novel targeted therapies with chemoradiotherapy in clinical trials is discussed, as well as new and promising biomarkers to test drug activity (Herrera, 2008).

\section{Overexpression of epidermal growth factor type-1 receptor (EGF-R1) in cervical cancer: A possible therapeutic target for cervical carcinoma}

Overexpression of epidermal growth factor type-1 receptor (EGF-R1) has been found in more than $70 \%$ of carcinomas of the cervix.

\subsection{Cetuximab}

Cetuximab, a monoclonal antibody, binds specifically to the epidermal growth factor receptor (EGFR) and competitively inhibits the binding of epidermal growth factor and other ligands. Cetuximab is clinically approved for the treatment of EGFR-expressing metastatic colorectal cancer and advanced head and neck cancer. Cetuximab might be a novel and attractive therapeutic strategy in patients harboring chemotherapy-resistant, recurrent, or metastatic cervical cancer. 
As anti-EGFR monoclonal antibodies sensitise tumours, Cetuximab's toxicity plus chemoradiation on cervical cancer cells which express different EGFR levels was investigated. This study showed that Cetuximab combined with chemoradiation, trastuzumab or MAPK inhibitors has useful applications for cervical cancer treatment, independently of EGFR expression (Meira, 2009).

In cervical cancer the expression of EGFR is reported in up to $85 \%$ of the tumour cells. Therefore, Cetuximab monotherapy could be a new option in the treatment of patients with advanced cervical cancer.

In a retrospective study, 5 patients with stage IIB to IVB cervical cancer were treated with Cetuximab monotherapy $250 \mathrm{mg} / \mathrm{m}^{2}$ weekly after an initial loading dose of $400 \mathrm{mg} / \mathrm{m}^{2}$ as third- to fifth-line therapy. Only one patient (20\%) had a stable disease, and the other four a progressive disease. Four out of five patients $(80 \%)$ developed an acneiform rash. The median survival time was 8.6 months.

This study showed no advantage in the treatment with Cetuximab monotherapy in patients with advanced cervical cancer. Further studies are necessary to evaluate the significance of Cetuximab in the treatment of advanced cervical cancer (Hertlein, 2011).

In a phase II GINECO trial, patients with advanced cervical squamous cell cancer or adenocarcinoma and at least one measurable target received intravenous cisplatin $50 \mathrm{mg} / \mathrm{m}^{2}$ on day 1 plus topotecan $0.75 \mathrm{mg} / \mathrm{m}^{2} /$ day from days 1 to 3 every 3 weeks combined with cetuximab (initial dose of $400 \mathrm{mg} / \mathrm{m}^{2}$ followed by subsequent weekly dose of $250 \mathrm{mg} / \mathrm{m}^{2}$ ).

Nineteen out of the 44 planned patients were accrued before the study was stopped early due to excessive toxicity. The most frequent adverse event was severe myelosuppression. The main grades 3-4 non-hematologic toxicities were infection (39\%) and febrile neutropenia $(28 \%)$, skin reactions $(22 \%)$, renal toxicity $(11 \%)$, and pulmonary embolism $(11 \%)$. Five $(28 \%)$ patients died during the treatment including 3 deaths related to treatment toxicity. Six $(32 \%)$ evaluable patients achieved a partial response. The median times of progression free survival and overall survival were 172 and 220 days, respectively.

In this phase II trial, the combination cisplatin - topotecan - cetuximab induced a high rate of serious adverse and/or fatal events at standard dose and schedule (Kurtz, 2009).

\subsection{Erlotinib}

A phase I trial was aimed to determine the maximum tolerated dose and related toxicity of erlotinib when administered concurrently with cisplatin and radiotherapy (CRT) for patients with locally advanced cervical squamous cell cancer. There was three cohorts of at least three patients receiving escalating doses of erlotinib combined with cisplatin $\left(40 \mathrm{mg} / \mathrm{m}^{2}\right.$, weekly, 5 cycles) and radiotherapy (external beam 4,500 cGy in 25 fractions, followed by 4 fractions $/ 600$ cGy/weekly of brachytherapy) in squamous cell cervical carcinoma patients, stage IIB to IIIB.

Fifteen patients were enrolled, 3 at dose level (DL) $50 \mathrm{mg}$, 4 at DL $100 \mathrm{mg}$, and 8 at DL 150 mg. Patients presented median age 47 (36-59), stage IIB (46.2\%) and IIIB (53.8\%). Overall, erlotinib+CRT was well-tolerated. Three patients did not complete the planned schedule. One patient at DL $100 \mathrm{mg}$ withdrew informed consent due to grade 2 rash; at DL $150 \mathrm{mg}, 1$ patient presented Raynaud's Syndrome and had C interrupted, and another patient presented grade 4 hepatotoxicity. The latter was interpreted as dose limiting toxicity and a 
new cohort of $150 \mathrm{mg}$ was started. No further grade 4 toxicity occurred. Grade 3 toxicity occurred in 6 cases: diarrhea in 3 patients, rash in 2 patients, and leukopenia in 1 patient. Erlotinib +CRT did not lead to limiting in-field toxicity.

This study showed that erlotinib + CRT was feasible to locally advanced squamous cell cervical cancer and was well tolerated. The maximum tolerated dose has been defined as 150 mg (Nogueira-Rodrigues, 2008).

A phase II trial of erlotinib in recurrent squamous cell carcinoma of the cervix was aimed to determine the proportion of patients with tumor response, the proportion who survived progression-free for at least 6 months and the frequency and severity of toxicities of patients with recurrent squamous cell carcinoma of the uterine cervix treated with erlotinib. This multicenter, open-label, single-arm trial evaluated the toxicity and efficacy of oral erlotinib at an initial dosage of $150 \mathrm{mg}$ daily until progressive disease or adverse effects prohibited further therapy.

Twenty-eight patients with squamous cell carcinoma were enrolled and 25 patients were evaluable. There were no objective responses, with 4 (16\%) patients achieving stable disease; only 1 patient had a progression-free survival of 6 months (4\%) or more. Erlotinib was well tolerated, with the most common drug-related adverse events being gastrointestinal toxicities, fatigue, and rash. This study showed that erlotinib was inactive as monotherapy in patients with recurrent squamous cell carcinoma of the uterine cervix (Schilder, 2009).

\subsection{Gefitinib}

Evidence suggests the epidermal growth factor receptor (EGFR) is expressed at moderate to high levels in cervical carcinomas. A multicenter, open-label, non-comparative phase II trial (study 1839IL/0075) investigated whether gefitinib (IRESSA), an EGFR tyrosine kinase inhibitor, is a potential second- or third-line treatment option for patients with recurring locoregionally advanced or metastatic cervical cancer. This study evaluated the clinical outcomes of $500 \mathrm{mg} /$ day gefitinib.

Thirty patients with squamous-cell carcinoma or adenocarcinoma were recruited from six centers in France. Of these, 28 patients were evaluable for efficacy. Although there were no objective responses, six $(20 \%)$ patients experienced stable disease with a median duration of 111.5 days. Median time to progression was 37 days and median overall survival was 107 days. Disease control did not appear to correlate with levels of EGFR expression. Gefitinib was well tolerated, with the most common drug-related adverse events being skin and gastrointestinal toxicities.

This study showed that gefitinib has only minimal monotherapy activity in recurrent disease resistant to standard treatment. However, the observation that $20 \%$ of patients treated with gefitinib had stable disease may warrant further investigation (Goncalves, 2008).

\section{NF-KB inhibition}

Nuclear factor-kappa B (NF-kappaB) is a transcription factor that plays a critical role across many cellular processes including embryonic and neuronal development, cell proliferation, apoptosis, and immune responses to infection and inflammation. Dysregulation of NFkappaB signaling is associated with inflammatory diseases and certain cancers. 
Constitutive activation of NF-kappaB signaling has been found in some types of tumors including breast, colon, prostate, skin and lymphoid, hence therapeutic blockade of NFkappaB signaling in cancer cells provides an attractive strategy for the development of anticancer drugs.

Miller and al. indicated that many currently approved pharmaceuticals have previously unappreciated effects on NF-kappaB signaling, which may contribute to anticancer therapeutic effects. Comprehensive profiling of approved drugs provides insight into their molecular mechanisms, thus providing a basis for drug repurposing (Miller, 2010).

Kima and al. suggested that the HPV 16 E5 oncoprotein mediates cervical carcinogenesis at least in part via upregulation of COX-2 expression through NF-kappaB and AP-1, with NFkappaB playing a larger role (Kim, 2009).

NF-kappaB activation is known to reduce the efficiency of chemotherapy in cancer treatment. Ursolic acid, a minimally toxic compound, has shown the capability to inhibit NF-kappaB activation in living cells. Li and al. investigated the effects and mechanisms of NF-kappaB inhibition by ursolic acid on chemotherapy treatment (Taxol or cisplatin) of cancerBy supplementing chemotherapy with minimally toxic ursolic acid, it is possible to improve the efficacy of cancer treatment by significantly reducing the necessary drug dose without sacrificing the treatment results $(\mathrm{Li}, 2010)$.

This recent studies suggested that NF-kappaB may contribute to the resistance of human cervical cancer cells to cisplatin and highlight the potential use of combination therapy involving cisplatin and NF-kappaB inhibitors.

\section{Cyclooxygenase-2 inhibitor}

Evidence from clinical and preclinical studies indicates that COX-2-derived prostaglandins participate in carcinogenesis, inflammation, immune response suppression, apoptosis inhibition, angiogenesis, and tumor cell invasion and metastasis. Clinical trial results have demonstrated that selective inhibition of COX-2 can alter the development and the progression of cancer. In animal models, selective inhibition of COX-2 activity is associated with the enhanced radiation sensitivity of tumors without appreciably increasing the effects of radiation on normal tissue, and preclinical evidence suggests that the principal mechanism of radiation potentiation through selective COX-2 inhibition is the direct increase in cellular radiation sensitivity and the direct inhibition of tumor neovascularization (Choy, 2003).

A phase I-II accrued 31 women with locally advanced cervical cancer to receive celecoxib $400 \mathrm{mg}$ by mouth twice per day for 2 weeks before and during chemoradiotherapy (CRT). Tumor oxygenation (HP(5)) and interstitial fluid pressure (IFP) were measured before and 2 weeks after celecoxib administration alone. The median follow-up time was 2.7 years.

The most common acute G3/4 toxicities were hematologic and gastrointestinal largely attributed to chemotherapy. Late G3/4 toxicity was seen in 4 of 31 patients, including fistulas in 3 patients. Within the first year of follow-up, 25 of 31 patients achieved complete response (CR), of whom 20 remained in CR at last follow-up. After 2 weeks of celecoxib administration before CRT, the median IFP decreased slightly, whereas HP(5) did not change significantly. No significant associations were seen between changes in HP(5) or IFP and response to treatment. 
Celecoxib in combination with definitive CRT is associated with acceptable acute toxicity, but higher than expected late complications. Celecoxib is associated with a modest reduction in the angiogenic biomarker IFP, but this does not correspond with tumor response (Herrera, 2007).

A phase II study determined the efficacy, patterns of initial failure and treatment-related acute toxicity rates in patients with locally advanced cancer of the cervix treated by oral celecoxib, intravenous cisplatin and 5-fluorouracil, and concurrent pelvic radiation therapy in patients with locally advanced cancer of the cervix.

Eligible patients included FIGO Stage IIB-IVA or patients with FIGO Stage IB through IIA with biopsy proven pelvic node metastases or tumor size $>$ or $=5 \mathrm{~cm}$. Celecoxib was prescribed at a dose of $400 \mathrm{mg}$ twice daily for 1 year beginning on the first day of radiotherapy. Cisplatin (75 $\left.\mathrm{mg} / \mathrm{m}^{2}\right)$ and $5-\mathrm{FU}\left(1 \mathrm{~g} / \mathrm{m}^{2}\right.$ for 4 days) were administered every 3 weeks times 3 . A total of 84 patients were accrued, of whom 78 were eligible and 77 were evaluable for toxicity.

At 2 years, the estimated disease-free survival and overall survival rate for patients with advanced cervical cancer who underwent a combination of chemoradiotherapy and celecoxib treatment was $69 \%$ and $83 \%$, respectively. Of the 78 patients, 24 had treatment failure and, of those patients, 18 had a component of locoregional failure as a site of first failure (Gaffney, 2007a). Toxicities were observed in the following areas: blood/bone marrow (16), gastrointestinal (14), pain (7), renal/genitourinary (6), cardiovascular (3), hemorrhage (1), and neurologic (1). For the first 75 evaluable patients, a toxicity failure was identified in 36 patients for a rate of $48 \%$ (Gaffney, 2007b).

Thus, locoregional control continues to be problematic after chemoradiotherapy (Gaffney, 2007a). Celecoxib at $400 \mathrm{mg}$ twice daily together with concurrent cisplatin and 5-FU and pelvic radiotherapy has a high incidence of acute toxicities. The most frequent toxicities were hematologic (Gaffney, 2007b).

Previous data demonstrate an association between cyclooxygenase activity and development of cervical cancer. A review investigated the role of cyclooxygenase- 2 (COX-2) in the development of cervical cancer and potential therapeutic options targeting this pathway.

Studies in vivo and in vitro confirm the role of COX-2 in the development of cervical cancer. In addition, COX-2 overexpression is associated with an increase in angiogenesis markers. Clinical correlation found that COX-2 overexpression in cervical cancer patients is a poor prognostic marker associated with increased risk for recurrent or metastatic disease. Despite early promise, two phase II trials in use of specific COX-2 inhibitors as radio-sensitizers in locally advanced cervical cancer demonstrated increased toxicity with no change in therapeutic effect. Results of studies using COX-2 inhibitors in pre-invasive cervical disease are encouraging (Young, 2008).

Jung and al. evaluated 67 FIGO stage IB2-IVA cervical cancer patients treated with cisplatinbased chemoradiotherapy (CCRT). The study group included patients who received rofecoxib $(\mathrm{N}=30)$ and the control group included patients who received CCRT only $(\mathrm{N}=37)$.

There were no significant differences in toxicity between the two groups. The most common acute grade 3/4 toxicity was neutropenia. Grade 3/4 late toxicity was observed in 2 patients in the study group and 3 in the control group. There was no treatment-related deaths in either group. Six patients in the study group had treatment failure. In the control group, 6 
patients experienced treatment failure. There were no differences in progression-free and overall survival between the 2 groups.

This data indicated that rofecoxib, at a dose of $25 \mathrm{mg}$ twice daily, has acceptable acute toxicity as a radiosensitizer during CCRT. Although rofecoxib was not efficacious as a radiosensitizer in the present study, the benefit of rofecoxib as a radiosensitizer should be further evaluated in a prospective study (Jung, 2009).

\section{Anti-angiogenesis agents}

Angiogenesis is central to cervical cancer development and progression. The dominant role of angiogenesis in cervical cancer seems to be directly related to HPV inhibition of p53 and stabilization of HIF-1 alpha, both of which increase vascular endothelial growth factor (VEGF).

\subsection{Bevacizumab}

Bevacizumab binding and subsequent inactivation of VEGF seem to shrink cervical tumors and delay progression without appreciable toxicity, and are therefore being studied in a Gynecologic Oncology Group (GOG) phase III trial. Other intracellular tyrosine kinase inhibitors (TKIs) of angiogenesis such as pazopanib are also encouraging, especially in lieu of their oral administration (Monk, 2010).

The Gynecologic Oncology Group (GOG) conducted a phase II trial to assess the efficacy and tolerability of bevacizumab. Eligible patients had recurrent cervical cancer, measurable disease, and GOG performance status < or $=2$. Treatment consisted of bevacizumab $15 \mathrm{mg} / \mathrm{kg}$ intravenously every 21 days until disease progression or prohibitive toxicity. Forty-six patients were enrolled. Grade 3 or 4 adverse events at least possibly related to bevacizumab included hypertension, thrombo-embolism, gastro-intestinal, anemia, other cardiovascular, vaginal bleeding, neutropenia, and fistula. One grade 5 infection was observed. Eleven patients $(23.9 \%)$ survived progression free for at least 6 months, and five patients $(10.9 \%)$ had partial responses. The median response duration was 6.21 months. The median PFS and overall survival times were 3.40 months and 7.29 months, respectively (Monk, 2009).

A retrospective analysis of women with recurrent cervical cancer treated with bevacizumab combination therapy was performed. Six patients were identified. The patients had a median of 3 prior regimens. All of the patients had multisite, metastatic disease. The combination regimen included IV 5-fluorouracil in 5 (83\%) patients and capecitabine in one $(17 \%)$ subject. Treatment was well tolerated. Grade 4 toxicity occurred in one patient who developed neutropenic sepsis. Clinical benefit (CR, PR, or SD) was noted in $67 \%$ of the subjects. This included 1 (17\%) complete response, 1 (17\%) partial response and two (33\%) patients with stable disease. The median time to progression for the four women who demonstrated clinical benefit was 4.3 months (Wright, 2006).

\subsection{Pazopanib}

Pazopanib is an oral multi-targeted TKI that binds to the vascular endothelial growth factor receptor (VEGFR), platelet-derived growth factor receptor (PDGFR) and c-Kit or epidermal growth factor receptor (EGFR) and human epidermal growth factor receptor 2 (HER2/neu), responsible for angiogenesis, tumor growth and cell survival. 
Pazopanib exhibited in vivo and in vitro activity against tumor growth and, in early clinical trials, was well tolerated with the main side effects being hypertension, fatigue and gastrointestinal disorders. Pazopanib showed clinical activity in several tumors including renal cell cancer (RCC), breast cancer, soft tissue sarcoma, thyroid cancer, hepatocellular cancer and cervical cancer (Schutz, 2011).

A phase II open-label study compared pazopanib or lapatinib monotherapy with pazopanib plus lapatinib combination therapy in patients with advanced and recurrent cervical cancer.

Patients with measurable stage IVB persistent/recurrent cervical carcinoma not amenable to curative therapy and at least one prior regimen in the metastatic setting were randomly assigned in a ratio of 1:1:1 to pazopanib at $800 \mathrm{mg}$ once daily, lapatinib at 1,500 $\mathrm{mg}$ once daily, or lapatinib plus pazopanib combination therapy (lapatinib at 1,000 mg plus pazopanib at 400 $\mathrm{mg}$ once daily or lapatinib at 1,500 $\mathrm{mg}$ plus pazopanib at $800 \mathrm{mg}$ once daily).

Of 230 patients enrolled, 152 were randomly assigned to the monotherapy arms: pazopanib $(\mathrm{n}=74)$ or lapatinib $(\mathrm{n}=78)$. Most patients $(62 \%)$ had recurrent cancer. Pazopanib improved PFS (hazard ratio [HR], 0.66; $90 \% \mathrm{CI}, 0.48$ to $0.91 ; \mathrm{P}=.013$ ) and OS (HR, $0.67 ; 90 \% \mathrm{CI}, 0.46$ to 0.99; $\mathrm{P}=.045)$. Median OS was 50.7 weeks and 39.1 weeks and RRs were $9 \%$ and $5 \%$ for pazopanib and lapatinib, respectively. The only grade $3 \mathrm{AE}>10 \%$ was diarrhea $(11 \%$ pazopanib and 13\% lapatinib). Grade 4 AEs were 9\% (lapatinib) and 12\% (pazopanib).

This study confirms the activity of antiangiogenesis agents in advanced and recurrent cervical cancer and demonstrates the benefit of pazopanib based on the prolonged PFS and favorable toxicity profile. Further study of angiogenesis and its inhibition are ongoing (Monk, 2010).

\section{References}

Choy H, Milas L (2003). Enhancing radiotherapy with cyclooxygenase-2 enzyme inhibitors: a rational advance? J Natl Cancer Inst. Vol 1;95(19):1440-52.

Gaffney DK, Winter K, Dicker AP, Miller B, Eifel PJ, Ryu J, Avizonis V, Fromm M, Small W, Greven K (2007). Efficacy and patterns of failure for locally advanced cancer of the cervix treated with celebrex (celecoxib) and chemoradiotherapy in RTOG 0128. Int J Radiat Oncol Biol Phys. Vol 1;69(1):111-7.

Gaffney DK, Winter K, Dicker AP, Miller B, Eifel PJ, Ryu J, Avizonis V, Fromm M, Greven K (2007). A Phase II study of acute toxicity for Celebrex (celecoxib) and chemoradiation in patients with locally advanced cervical cancer: primary endpoint analysis of RTOG 0128. Int J Radiat Oncol Biol Phys. Vol 1;67(1):104-9.

Goncalves A, Fabbro M, Lhommé C, Gladieff L, Extra JM, Floquet A, Chaigneau L, Carrasco AT, Viens P (2008). A phase II trial to evaluate gefitinib as second- or third-line treatment in patients with recurring locoregionally advanced or metastatic cervical cancer. Gynecol Oncol. Vol 108(1):42-6.

Herrera FG, Chan P, Doll C, Milosevic M, Oza A, Syed A, Pintilie M, Levin W, Manchul L, Fyles A (2007). A prospective phase I-II trial of the cyclooxygenase-2 inhibitor celecoxib in patients with carcinoma of the cervix with biomarker assessment of the tumor microenvironment. Int J Radiat Oncol Biol Phys. Vol 1;67(1):97-103.

Herrera FG, Vidal L, Oza A, Milosevic M, Fyles A (2008). Molecular targeted agents combined with chemo-radiation in the treatment of locally advanced cervix cancer. Rev Recent Clin Trials. Vol 3(2):111-20. 
Hertlein L, Lenhard M, Kirschenhofer A, Kahlert S, Mayr D, Burges A, Friese K (2011). In a retrospective study, Cetuximab monotherapy was applied in advanced cervical cancer. Arch Gynecol Obstet. Vol 283(1):109-13.

Jung YW, Lee SH, Paek JH, Nam EJ, Kim SW, Kim JH, Kim JW, Kim YT (2009). Acute toxicity of cyclooxygenase-2 inhibitor rofecoxib as a radiosensitizer for concurrent chemoradiation in the treatment of uterine cervical cancer. J Gynecol Oncol. Vol 20(3):151-7.

Kim SH, Oh JM, No JH, Bang YJ, Juhnn YS, Song YS (2009). Involvement of NF-kappaB and AP-1 in COX-2 upregulation by human papillomavirus 16 E5 oncoprotein. Carcinogenesis. Vol 30(5):753-7.

Kurtz JE, Hardy-Bessard AC, Deslandres M, Lavau-Denes S, Largillier R, Roemer-Becuwe C, Weber B, Guillemet C, Paraiso D, Pujade-Lauraine E (2009). Cetuximab, topotecan and cisplatin for the treatment of advanced cervical cancer: A phase II GINECO trial. Gynecol Oncol. Vol 113(1):16-20.

Li Y, Xing D, Chen Q, Chen WR (2010). Enhancement of chemotherapeutic agent-induced apoptosis by inhibition of NF-kappaB using ursolic acid. Int $J$ Cancer. Vol 15;127(2):462-73.

Meira DD, de Almeida VH, Mororó JS, Nóbrega I, Bardella L, Silva RL, Albano RM, Ferreira CG (2009). Combination of cetuximab with chemoradiation, trastuzumab or MAPK inhibitors: mechanisms of sensitisation of cervical cancer cells. $\mathrm{Br} J$ Cancer. Vol 1;101(5):782-91.

Miller SC, Huang R, Sakamuru S, Shukla SJ, Attene-Ramos MS, Shinn P, Van Leer D, Leister W, Austin CP, Xia M (2010). Identification of known drugs that act as inhibitors of NF-kappaB signaling and their mechanism of action. Biochem Pharmacol. Vol 1;79(9):1272-80.

Monk BJ, Sill MW, Burger RA, Gray HJ, Buekers TE, Roman LD (2009). Phase II trial of bevacizumab in the treatment of persistent or recurrent squamous cell carcinoma of the cervix: a gynecologic oncology group study. J Clin Oncol. Vol 1;27(7):1069-74.

Monk BJ, Mas Lopez L, Zarba JJ, Oaknin A, Tarpin C, Termrungruanglert W, Alber JA, Ding J, Stutts MW, Pandite LN (2010). Phase II, open-label study of pazopanib or lapatinib monotherapy compared with pazopanib plus lapatinib combination therapy in patients with advanced and recurrent cervical cancer. J Clin Oncol. Vol 1;28(22):3562-9.

Monk BJ, Willmott LJ, Sumner DA (2010). Anti-angiogenesis agents in metastatic or recurrent cervical cancer. Gynecol Oncol. Vol 116(2):181-6.

Nogueira-Rodrigues A, do Carmo CC, Viegas C, Erlich F, Camisão C, Fontão K, Lima R, Herchenhorn D, Martins RG, Moralez GM, Small IA, Ferreira CG (2008). Phase I trial of erlotinib combined with cisplatin and radiotherapy for patients with locally advanced cervical squamous cell cancer. Clin Cancer Res. Vol 1;14(19):6324-9.

Schilder RJ, Sill MW, Lee YC, Mannel R (2009). A phase II trial of erlotinib in recurrent squamous cell carcinoma of the cervix: a Gynecologic Oncology Group Study. Int J Gynecol Cancer. Vol 19(5):929-33.

Schutz FA, Choueiri TK, Sternberg CN (2011). Pazopanib: Clinical development of a potent anti-angiogenic drug. Crit Rev Oncol Hematol. Vol 77(3):163-71.

Wright JD, Viviano D, Powell MA, Gibb RK, Mutch DG, Grigsby PW, Rader JS (2006). Bevacizumab combination therapy in heavily pretreated, recurrent cervical cancer. Gynecol Oncol. Vol 103(2):489-93.

Young JL, Jazaeri AA, Darus CJ, Modesitt SC (2008). Cyclooxygenase-2 in cervical neoplasia: a review. Gynecol Oncol. Vol 109(1):140-5. 


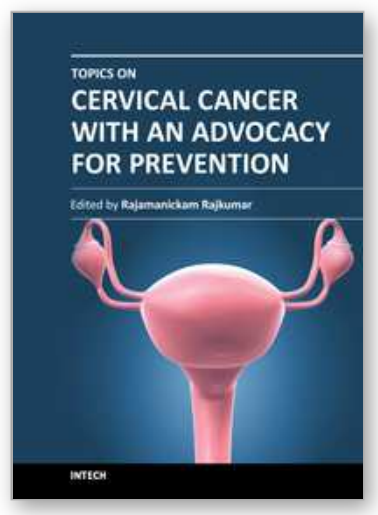

\author{
Topics on Cervical Cancer With an Advocacy for Prevention \\ Edited by Dr. R. Rajamanickam
}

ISBN 978-953-51-0183-3

Hard cover, 284 pages

Publisher InTech

Published online 02, March, 2012

Published in print edition March, 2012

Cervical Cancer is one of the leading cancers among women, especially in developing countries. Prevention and control are the most important public health strategies. Empowerment of women, education, "earlier" screening by affordable technologies like visual inspection, and treatment of precancers by cryotherapy/ LEEP are the most promising interventions to reduce the burden of cervical cancer.Dr Rajamanickam Rajkumar had the privilege of establishing a rural population based cancer registry in South India in 1996, as well as planning and implementing a large scale screening program for cervical cancer in 2000 . The program was able to show a reduction in the incidence rate of cervical cancer by $25 \%$, and reduction in mortality rate by $35 \%$. This was the greatest inspiration for him to work on cerrvical cancer prevention, and he edited this book to inspire others to initiate such programs in developing countries. InTech - Open Access Publisher plays a major role in this crusade against cancer, and the authors have contributed to it very well.

\title{
How to reference
}

In order to correctly reference this scholarly work, feel free to copy and paste the following:

Magali Provansal, Maria Cappiello, Frederique Rousseau, Anthony Goncalves and Patrice Viens (2012). New Therapeutic Targets, Topics on Cervical Cancer With an Advocacy for Prevention, Dr. R. Rajamanickam (Ed.), ISBN: 978-953-51-0183-3, InTech, Available from: http://www.intechopen.com/books/topics-on-cervicalcancer-with-an-advocacy-for-prevention/new-therapeutic-targets

\section{INTECH}

open science | open minds

\author{
InTech Europe \\ University Campus STeP Ri \\ Slavka Krautzeka 83/A \\ 51000 Rijeka, Croatia \\ Phone: +385 (51) 770447 \\ Fax: +385 (51) 686166 \\ www.intechopen.com
}

\author{
InTech China \\ Unit 405, Office Block, Hotel Equatorial Shanghai \\ No.65, Yan An Road (West), Shanghai, 200040, China \\ 中国上海市延安西路65号上海国际贵都大饭店办公楼 405 单元 \\ Phone: +86-21-62489820 \\ Fax: $+86-21-62489821$
}


(C) 2012 The Author(s). Licensee IntechOpen. This is an open access article distributed under the terms of the Creative Commons Attribution 3.0 License, which permits unrestricted use, distribution, and reproduction in any medium, provided the original work is properly cited. 